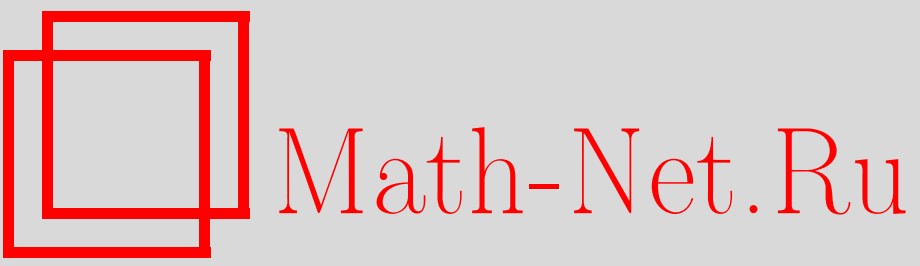

О. И. Морозов, Свойства интегрируемости четырехмерного уравнения универсальной иерархии, Итоги науки и техн. Сер. Соврем. мат. и ее прил. Темат. обз., 2019, том 169, 48-55

DOI: https://doi.org/10.36535/0233-6723-2019-169-48-55

Использование Общероссийского математического портала Math-Net.Ru подразумевает, что вы прочитали и согласны с пользовательским соглашением

http: //www.mathnet.ru/rus/agreement

Параметры загрузки:

IP: 54.162 .85 .209

26 апреля 2023 г., 13:43:45 


\title{
СВОЙСТВА ИНТЕГРИРУЕМОСТИ ЧЕТЫРЕХМЕРНОГО УРАВНЕНИЯ УНИВЕРСАЛЬНОЙ ИЕРАРХИИ
}

\author{
(c) 2019 г. $\quad$ О. И. МОРОЗОВ
}

\begin{abstract}
Аннотация. Рассмотрены свойства, связанные с интегрируемостью четырехмерного уравнения универсальной иерархии. В частности, изучена структура алгебры его локальных симметрий и показано, что вторая группа экзотических когомологий этой алгебры нетривиальна. Доказано, что спектральный параметр в известном накрытии этого уравнения является неустранимым. Найдена тень нелокальной симметрии; с ее помощью построен оператор рекурсии. Действие оператора рекурсии на некоторые локальные симметрии производит новые неабелевы накрытия изучаемого уравнения.
\end{abstract}

Ключевые слова: интегрируемое дифференциальное уравнение, дифференциальное накрытие, симметрия, оператор рекурсии.

\section{INTEGRABILITY PROPERTIES OF THE FOUR-DIMENSIONAL EQUATION OF THE UNIVERSAL HIERARCHY}

\section{(c) 2019 O. I. MOROZOV}

\begin{abstract}
Properties associated with the integrability of the four-dimensional equation of the universal hierarchy are considered. In particular, the structure of the algebra of its local symmetries is studied. We show that the second group of exotic cohomologies of this algebra is nontrivial. We prove that the spectral parameter in the well-known covering of this equation is irremovable. A shadow of nonlocal symmetry was found; using it, we construct the recursion operator. The action of the recursion operator on some local symmetries generates new non-Abelian coverings of the equation.
\end{abstract}

Keywords and phrases: integrable differential equation, differential covering, symmetry, recursion operator.

AMS Subject Classification: 58J70, 58J72, 35A30

1. Введение. Рассмотрим четырехмерное нелинейное дифференциальное уравнение

$$
u_{z z}=u_{t x}+u_{x} u_{y z}-u_{z} u_{x y}
$$

введенное в [5]. Задаваемая подстановкой $u_{t}=0$ трехмерная редукция уравнения (1) дает уравнение универсальной иерархии (см. $[3,18])$

$$
u_{z z}=u_{x} u_{y z}-u_{z} u_{x y}
$$

поэтому мы будем называть уравнение (1) четырехмерным уравнением универсальной иерархии. В [26] для уравнения (1) было найдено дифференциальное накрытие

$$
\left\{\begin{array}{l}
q_{t}=\lambda^{2} q_{x}+\left(\lambda u_{x}+u_{z}\right) q_{y} \\
q_{z}=\lambda q_{x}+u_{x} q_{y} .
\end{array}\right.
$$


В данной статье мы изучаем свойства, связанные с интегрируемостью уравнения (1), в частности, анализируем структуру алгебры его локальных симметрий и показываем, что вторая группа экзотических когомологий этой алгебры нетривиальна. Мы доказываем, что параметр $\lambda$ в накрытии (2) является неустранимым, а затем находим тень нелокальной симметрии и используем ее для построения оператора рекурсии, который является важным атрибутом интегрируемого дифференциального уравнения. Мы показываем, что действие оператора рекурсии на некоторые локальные симметрии производит новые накрытия изучаемого уравнения.

\section{2. Предварительные сведения.}

2.1. Симметрии и накрытия. Изложение в этом разделе близко следует статьям [11,12] (см. также [14,15]). Пусть $\pi: \mathbb{R}^{n} \times \mathbb{R}^{m} \rightarrow \mathbb{R}^{n}, \pi:\left(x^{1}, \ldots, x^{n}, u^{1}, \ldots, u^{m}\right) \mapsto\left(x^{1}, \ldots, x^{n}\right)$ является тривиальным расслоением, а $J^{\infty}(\pi)$ является расслоением джетов его сечений бесконечного порядка. Локальные координаты на $J^{\infty}(\pi)$ имеют вид $\left(x^{i}, u^{\alpha}, u_{I}^{\alpha}\right)$, где $I=\left(i_{1}, \ldots, i_{n}\right)$ - мультииндексы, и для любого локального сечения $f: \mathbb{R}^{n} \rightarrow \mathbb{R}^{n} \times \mathbb{R}^{m}$ расслоения $\pi$ соответствующий бесконечный джет $j_{\infty}(f)$ является таким сечением $j_{\infty}(f): \mathbb{R}^{n} \rightarrow J^{\infty}(\pi)$, что

$$
u_{I}^{\alpha}\left(j_{\infty}(f)\right)=\frac{\partial^{\# I} f^{\alpha}}{\partial x^{I}}=\frac{\partial^{i_{1}+\cdots+i_{n}} f^{\alpha}}{\left(\partial x^{1}\right)^{i_{1}} \ldots\left(\partial x^{n}\right)^{i_{n}}} .
$$

Положим $u^{\alpha}=u_{(0, \ldots, 0)}^{\alpha}$. Кроме того, в случае $n=4, m=1$ обозначим $x^{1}=t, x^{2}=x, x^{3}=y$, $x^{4}=z$ и $u_{(i, j, k, l)}^{1}=u_{t \ldots t x \ldots x y . \ldots y z \ldots z}$, где $t, x, y, z$ встречаются $i, j, k, l$ раз соответственно.

Векторные поля

$$
D_{x^{k}}=\frac{\partial}{\partial x^{k}}+\sum_{\# I \geqslant 0} \sum_{\alpha=1}^{m} u_{I+1_{k}}^{\alpha} \frac{\partial}{\partial u_{I}^{\alpha}}, \quad k \in\{1, \ldots, n\},
$$

где $\left(i_{1}, \ldots, i_{k}, \ldots, i_{n}\right)+1_{k}=\left(i_{1}, \ldots, i_{k}+1, \ldots, i_{n}\right)$, называются полными производными. Они коммутируют на всем расслоении $J^{\infty}(\pi)$, т.е. $\left[D_{x^{i}}, D_{x^{j}}\right]=0$.

Эволюиионное дифферениирование, связанное с произвольной гладкой векторнозначной функцией $\varphi: J^{\infty}(\pi) \rightarrow \mathbb{R}^{m}$, является векторным полем

$$
\mathbf{E}_{\varphi}=\sum_{\# I \geqslant 0} \sum_{\alpha=1}^{m} D_{I}\left(\varphi^{\alpha}\right) \frac{\partial}{\partial u_{I}^{\alpha}}
$$

где $D_{I}=D_{\left(i_{1}, \ldots i_{n}\right)}=D_{x^{1}}^{i_{1}} \circ \cdots \circ D_{x^{n}}^{i_{n}}$.

Система дифференциальных уравнений в частных производных (ДУ) $F_{r}\left(x^{i}, u_{I}^{\alpha}\right)=0, \# I \leqslant s$, $r \in\{1, \ldots, R\}$, порядка $s \geqslant 1$ с $R \geqslant 1$, определяет подмногообразие

$$
\mathcal{E}=\left\{\left(x^{i}, u_{I}^{\alpha}\right) \in J^{\infty}(\pi) \mid D_{K}\left(F_{r}\left(x^{i}, u_{I}^{\alpha}\right)\right)=0, \# K \geqslant 0\right\} \subset J^{\infty}(\pi) .
$$

Функция $\varphi: J^{\infty}(\pi) \rightarrow \mathbb{R}^{m}$ называется генератором инфинитезимальной симметрии, или просто симметрией уравнения $\mathcal{E}$, если $\mathbf{E}_{\varphi}(F)=0$ на $\mathcal{E}$. Симметрия $\varphi$ является решением определяющей системь

$$
\ell_{\mathcal{E}}(\varphi)=0,
$$

где $\ell_{\mathcal{E}}=\left.\ell_{F}\right|_{\mathcal{E}}$, с матричным дифференциальным оператором

$$
\ell_{F}=\left(\sum_{\# I \geqslant 0} \frac{\partial F_{r}}{\partial u_{I}^{\alpha}} D_{I}\right)
$$

Алгебра симметрий $\operatorname{sym}(\mathcal{E})$ состоит из решений системы (4). Скобка Якоби

$$
\{\varphi, \psi\}=\mathbf{E}_{\varphi}(\psi)-\mathbf{E}_{\psi}(\varphi)
$$

определяет на $\operatorname{sym}(\mathcal{E})$ структуру алгебры Ли над $\mathbb{R}$. Алгебра контактных симметрий $\operatorname{sym}_{0}(\mathcal{E})$ является подалгеброй Ли в $\operatorname{sym}(\mathcal{E})$, определенной пересечением $\operatorname{sym}(\mathcal{E}) \cap J^{1}(\pi)$. 
Рассмотрим $\mathcal{W}=\mathbb{R}^{\infty}$ с координатами $w^{s}, s \in \mathbb{N} \cup\{0\}$. Локально (бесконечномерное) дифберенuиальное накрытие уравнения $\mathcal{E}$ является тривиальным расслоением $\tau: J^{\infty}(\pi) \times \mathcal{W} \rightarrow J^{\infty}(\pi)$, снабженным расширенными полными производными

$$
\widetilde{D}_{x^{k}}=D_{x^{k}}+\sum_{s=0}^{\infty} T_{k}^{s}\left(x^{i}, u_{I}^{\alpha}, w^{j}\right) \frac{\partial}{\partial w^{s}},
$$

удовлетворяющими условию $\left[\widetilde{D}_{x^{i}}, \widetilde{D}_{x^{j}}\right]=0$ для всех $i \neq j$ тогда и только тогда, когда $\left(x^{i}, u_{I}^{\alpha}\right) \in \mathcal{E}$. Определим частные производные переменных $w^{s}$ равенствами $w_{x^{k}}^{s}=\widetilde{D}_{x^{k}}\left(w^{s}\right)$. Это дает систему накрывающих уравнений

$$
w_{x^{k}}^{s}=T_{k}^{s}\left(x^{i}, u_{I}^{\alpha}, w^{j}\right) .
$$

Эта переопределенная система ДУ совместна тогда и только тогда, когда $\left(x^{i}, u_{I}^{\alpha}\right) \in \mathcal{E}$.

Двойственным образом, накрытие определяется формами Уолквиста-Эстабрука

$$
\omega^{s}=d w^{s}-\sum_{k=1}^{m} T_{k}^{s}\left(x^{i}, u_{I}^{\alpha}, w^{j}\right) d x^{k} .
$$

Обозначим через $\widetilde{\mathbf{E}}_{\varphi}$ результат подстановки $\widetilde{D}_{x^{k}}$ вместо $D_{x^{k}}$ в $(3)$. Тенъю нелокальной симметрии уравнения $\mathcal{E}$ в накрытии $\tau$ с расширенными полными производными (5), или $\tau$-тенъю, называется такая функция $\varphi \in C^{\infty}(\mathcal{E} \times \mathcal{W})$, что равенство

$$
\widetilde{\mathbf{E}}_{\varphi}(F)=0
$$

является следствием уравнений $D_{K}(F)=0$ и (6).

Нелокальной симметрией уравнения $\mathcal{E}$ в накрытии $\tau$, или $\tau$-симметрией, называется такое векторное поле

$$
\widetilde{\mathbf{E}}_{\varphi, A}=\widetilde{\mathbf{E}}_{\varphi}+\sum_{s=0}^{\infty} A^{s} \frac{\partial}{\partial w^{s}}
$$

с $A^{s} \in C^{\infty}(\mathcal{E} \times \mathcal{W})$, что $\varphi$ удовлетворяет (7) и

$$
\widetilde{D}_{k}\left(A^{s}\right)=\widetilde{\mathbf{E}}_{\varphi, A}\left(T_{k}^{s}\right)
$$

для $T_{k}^{s}$ из (5) (см. [2, гл. $\left.\left.6, \S 3.2\right]\right)$. В общем случае не каждая $\tau$-тень соответствует $\tau$-симметрии, так как уравнения (9) образуют препятствие для существования (8). Однако для любой $\tau$-тени $\varphi$ существует накрытие $\tau_{\varphi}$ и нелокальная $\tau_{\varphi}$-симметрия, для которой $\tau_{\varphi}$-тень совпадает с $\varphi$ (см. $[2$, Chap. 6, § 5.8]).

Оператором рекурсии $\mathcal{R}$ для уравнения $\mathcal{E}$ называется такое $\mathbb{R}$-линейное отображение, что для каждой (локальной или нелокальной) симметрии $\varphi$ уравнения $\mathcal{E}$ функция $\mathcal{R}(\varphi)$ является (локальной или нелокальной) симметрией $\mathcal{E}$.

Касательное накрытие уравнения $\mathcal{E}$ определяется следующим образом (см. [12]). Рассмотрим тривиальное расслоение $\sigma: J^{\infty}(\pi) \times \mathcal{V} \rightarrow J^{\infty}(\pi)$ с координатами $v_{I}^{\alpha}, \# I \geqslant 0$, на слое $\mathcal{V}$, снабженное расширенными полными производными

$$
\hat{D}_{x^{k}}=D_{x^{k}}+\sum_{\# I \geqslant 0} \sum_{\alpha=1}^{m} v_{I+1_{k}}^{\alpha} \frac{\partial}{\partial v_{I}^{\alpha}} .
$$

Для $\hat{D}_{I}=\hat{D}_{x^{1}}^{i_{1}} \circ \cdots \circ \hat{D}_{x^{n}}^{i_{n}}$ определим

$$
\hat{\ell}_{F}=\left(\sum_{\# I \geqslant 0} \frac{\partial F_{r}}{\partial u_{I}^{\alpha}} \hat{D}_{I}\right)
$$

и положим

$$
\mathcal{T}(\mathcal{E})=\left\{\left(x^{i}, u_{i}^{\alpha}, v_{I}^{\alpha}\right) \in J^{\infty}(\pi) \times \mathcal{V} \mid D_{K}\left(F\left(x^{i}, u_{I}^{\alpha}\right)\right)=0, \hat{D}_{K}\left(\hat{\ell}_{F}\left(v^{\alpha}\right)\right)=0, \# K \geqslant 0\right\} .
$$


Тогда касательное накрытие является ограничением $\sigma$ на $\mathcal{T}(\mathcal{E})$. Сечение $\varphi: \mathcal{E} \rightarrow \mathcal{T}(\mathcal{E})$ касательного накрытия явялется симметрией уравнения $\mathcal{E}$. Расширенные полные производные этого накрытия имеют вид $\widetilde{D}_{x^{k}}=\left.\hat{D}_{x^{k}}\right|_{\mathcal{T}(\mathcal{E})}$.

В соответствии с [19] оператор рекурсии для симметрий некоторого уравнения является автопреобразованием Бэклунда касательного накрытия этого уравнения (см. также детальное обсуждение в [13, Chap. 7]).

2.2. Экзотические когомологии алгебр Ли. Рассмотрим произвольную алгебру Ли $\mathfrak{g}$ над $\mathbb{R}$ и ее представление $\rho: \mathfrak{g} \rightarrow \operatorname{End}(V)$. Пусть $C^{k}(\mathfrak{g}, V)=\operatorname{Hom}\left(\Lambda^{k}(\mathfrak{g}), V\right)$ - пространство всех $k$-линейных кососимметрических отображений $(k \geqslant 1)$ из $\mathfrak{g}$ в $V$. Дифференциальный комплекс ШеваллеЭйленберга

$$
V=C^{0}(\mathfrak{g}, V) \stackrel{d}{\longrightarrow} C^{1}(\mathfrak{g}, V) \stackrel{d}{\longrightarrow} \ldots \stackrel{d}{\longrightarrow} C^{k}(\mathfrak{g}, V) \stackrel{d}{\longrightarrow} C^{k+1}(\mathfrak{g}, V) \stackrel{d}{\longrightarrow} \ldots
$$

порождается дифференциалом, определенным формулой

$$
\begin{aligned}
d \theta\left(X_{1}, \ldots, X_{k+1}\right)=\sum_{q=1}^{k+1}(-1)^{q+1} \rho\left(X_{q}\right)\left(\theta\left(X_{1}, \ldots, \hat{X}_{q}, \ldots, X_{k+1}\right)\right)+ & \\
& +\sum_{1 \leqslant p<q \leqslant k+1}(-1)^{p+q} \theta\left(\left[X_{p}, X_{q}\right], X_{1}, \ldots, \hat{X}_{p}, \ldots, \hat{X}_{q}, \ldots, X_{k+1}\right) .
\end{aligned}
$$

Когомологии комплекса $\left(C^{*}(\mathfrak{g}, V), d\right)$ называются когомологиями алгебры Ли $\mathfrak{g}$ с коэфбициентами в представлении $\rho$. Для тривиального представления $\rho_{0}: \mathfrak{g} \rightarrow \mathbb{R}, \rho_{0}: X \mapsto 0$, комплекс Шевалле-Эйленберга и его когомологии обозначаются $C^{*}(\mathfrak{g})$ и $H^{*}(\mathfrak{g})$ соответственно.

Рассмотрим алгебру Ли $\mathfrak{g}$ с нетривиальной первой группой когомологий $H^{1}(\mathfrak{g})$. Выберем ненулевую замкнутую 1-форму $\alpha$ на $\mathfrak{g}$. Для произвольного $\lambda \in \mathbb{R}$ определим новый дифференциал

$$
d_{\lambda \alpha}: C^{k}(\mathfrak{g}, \mathbb{R}) \rightarrow C^{k+1}(\mathfrak{g}, \mathbb{R}), \quad d_{\lambda \alpha} \theta=d \theta+\lambda \alpha \wedge \theta .
$$

Из условия $d \alpha=0$ следует, что $d_{\lambda \alpha}^{2}=0$. Когомологии комплекса

$$
C^{1}(\mathfrak{g}, \mathbb{R}) \stackrel{d_{\lambda \alpha}}{\longrightarrow} \ldots \stackrel{d_{\lambda \alpha}}{\longrightarrow} C^{k}(\mathfrak{g}, \mathbb{R}) \stackrel{d_{\lambda \alpha}}{\longrightarrow} C^{k+1}(\mathfrak{g}, \mathbb{R}) \stackrel{d_{\lambda \alpha}}{\longrightarrow} \ldots
$$

называются экзотическими когомологиями алгебры Ли $\mathfrak{g}$ и обозначаются $H_{\lambda \alpha}^{*}(\mathfrak{g})$.

3. Контактные симметрии четырехмерного уравнения универсальной иерархии. Прямые вычисления, проведенные с использованием пакета Jets (см. [4]), показывают, что алгебра Ли контактных симметрий $\mathfrak{s}=\operatorname{sym}_{0}(\mathcal{E})$ уравнения $(1)$ порождается функциями

$$
\begin{array}{ll}
\phi_{0}(A)=-A u_{y}+A_{y} u-A_{t} z, & \psi_{3}=-2 x u_{x}-z u_{z}+u, \\
\phi_{1}(A)=A, & \psi_{4}=-\frac{1}{2} z u_{x}-t u_{z}, \\
\psi_{1}=-t u_{t}+x u_{x}-u, & \psi_{5}=-u_{z}, \\
\psi_{2}=-u_{t}, & \psi_{6}=-u_{x}
\end{array}
$$

где $A=A(t, y)$ и $B=B(t, y)$ в последующих формулах - произвольные (гладкие) функции переменных $t$ и $y$. Коммутаторы этих генераторов задаются уравнениями

$$
\begin{array}{llll}
\left\{\phi_{0}(A), \phi_{0}(B)\right\}=\phi_{0}\left(A B_{y}-B A_{y}\right), \quad\left\{\phi_{0}(A), \phi_{1}(B)\right\}=\phi_{1}\left(A B_{y}-B A_{y}\right), & \left\{\phi_{1}(A), \phi_{1}(B)\right\}=0, \\
\left\{\psi_{1}, \phi_{0}(A)\right\}=\phi_{0}\left(t A_{t}\right), & \left\{\psi_{1}, \phi_{1}(A)\right\}=\phi_{1}\left(t A_{t}\right), & & \\
\left\{\psi_{2}, \phi_{0}(A)\right\}=\phi_{0}\left(A_{t}\right), & \left\{\psi_{2}, \phi_{1}(A)\right\}=\phi_{1}\left(A_{t}\right), & \left\{\psi_{1}, \psi_{2}\right\}=-\psi_{2}, & \left\{\psi_{1}, \psi_{4}\right\}=\psi_{4}, \\
\left\{\psi_{3}, \phi_{0}(A)\right\}=0, & \left\{\psi_{3}, \phi_{1}(A)\right\}=-\phi_{1}(A), & \left\{\psi_{1}, \psi_{6}\right\}=\psi_{6}, & \left\{\psi_{2}, \psi_{4}\right\}=\psi_{5}, \\
\left\{\psi_{4}, \phi_{0}(A)\right\}=-\phi_{1}\left(t A_{t}\right), & \left\{\psi_{4}, \phi_{1}(A)\right\}=0, & \left\{\psi_{3}, \psi_{4}\right\}=-\psi_{4}, & \left\{\psi_{3}, \psi_{5}\right\}=-\psi_{5}, \\
\left\{\psi_{5}, \phi_{0}(A)\right\}=-\phi_{1}\left(A_{t}\right), & \left\{\psi_{5}, \phi_{1}(A)\right\}=0, & \left\{\psi_{3}, \psi_{6}\right\}=-2 \psi_{6}, & \left\{\psi_{4}, \psi_{5}\right\}=-\frac{1}{2} \psi_{5}, \\
\left\{\psi_{6}, \phi_{0}(A)\right\}=0, & \left\{\psi_{6}, \phi_{1}(A)\right\}=0, &
\end{array}
$$


причем $\left\{\psi_{i}, \psi_{j}\right\}=0$ для всех остальных пар индексов $i<j$. Из этих равенств следует, что алгебра $\mathfrak{s}$ является полупрямой суммой $\mathfrak{s}=\mathfrak{s}_{\diamond} \ltimes \mathfrak{s}_{\infty}$ шестимерной алгебры $\mathfrak{s}_{\diamond}=\left\langle\psi_{k} \mid k \in\{1, \ldots, 6\}\right\rangle$ и бесконечномерной алгебры $\mathfrak{s}_{\infty}=\left\langle\phi_{0}(A), \phi_{1}(A) \mid A \in C^{\infty}\left(\mathbb{R}^{2}\right)\right\rangle$, которая, в свою очередь, изоморфна тензорному произведению $\mathbb{R}_{2}[h] \otimes \mathfrak{q}$ (коммутативной ассоциативной) алгебры усеченных многочленов $\mathbb{R}_{2}[h]=\mathbb{R}[h] /\left\langle h^{2}=0\right\rangle$ и алгебры Ли q векторных полей вида $A(t, y) \partial_{y}$.

Как было показано в [22-24], вторая группа экзотических когомологий алгебры симметрии дифференциального уравнения играет важную роль в изучении его свойств интегрируемости и представляет собой основу для построения его накрытий и интегрируемых расширений. В заключение этого раздела мы устанавливаем, что алебра симметрий $\mathfrak{s}$ уравнения (1) имеет нетривиальную вторую группу экзотических когомологий. Пусть $\beta_{1}, \ldots, \beta_{6}$ обозначают двойственные формы алгебры Ли $\mathfrak{s}_{\diamond}$, т.е. такие 1 -формы, что равенства $\beta_{i}\left(\psi_{j}\right)=\delta_{i j}$ выполнены при всех $i, j \in\{1, \ldots, 6\}$. Тогда структурные уравнения Маурера-Картана для этих форм имеют вид

$$
\begin{array}{ll}
d \beta_{1}=0, & d \beta_{4}=\left(\beta_{3}-\beta_{1}\right) \wedge \beta_{4}, \\
d \beta_{2}=\beta_{1} \wedge \beta_{2}, & d \beta_{5}=\beta_{3} \wedge \beta_{5}-\beta_{2} \wedge \beta_{4}, \\
d \beta_{3}=0, & d \beta_{6}=\left(2 \beta_{3}-\beta_{1}\right) \wedge \beta_{6}+\frac{1}{2} \beta_{4} \wedge \beta_{5} .
\end{array}
$$

Поэтому прямые вычисления дают

$$
H^{1}\left(\mathfrak{s}_{\diamond}\right)=\left\langle\psi_{1}, \psi_{3}\right\rangle
$$

при этом вторая группа экзотических когомологий отлична от $\{0\}$ в следующих случаях:

$$
H_{\mu_{1} \beta_{1}+\mu_{2} \beta_{3}}^{2}\left(\mathfrak{s}_{\diamond}\right)=\left\{\begin{array}{lll}
\left\langle\beta_{1} \wedge \beta_{2}, \beta_{2} \wedge \beta_{3}\right\rangle, & \mu_{1}=1, & \mu_{2}=0, \\
\left\langle\beta_{2} \wedge \beta_{4}\right\rangle, & \mu_{1}=1, & \mu_{2}=1, \\
\left\langle\beta_{1} \wedge \beta_{4}, \beta_{3} \wedge \beta_{4}\right\rangle, & \mu_{1}=-1, & \mu_{2}=1, \\
\left\langle\beta_{4} \wedge \beta_{6}\right\rangle, & \mu_{1}=-2, & \mu_{2}=3 .
\end{array}\right.
$$

Из структурных уравнений алгебры Ли $\mathfrak{s}$ следует, что 2-формы

$$
\beta_{1} \wedge \beta_{2}, \quad \beta_{2} \wedge \beta_{3}, \quad \beta_{2} \wedge \beta_{4}, \quad \beta_{1} \wedge \beta_{4}, \quad \beta_{3} \wedge \beta_{4}, \quad \beta_{4} \wedge \beta_{6}
$$

являются нетривиальными экзотическими коциклами всей алгебры $\mathfrak{s}$. Поэтому вторая группа экзотических когомологий алгебры симметрий уравнения (1) нетривиальна.

4. Неустранимость спектрального параметра. Покажем, что спектральный параметр $\lambda$ в накрытии (2) является неустранимым, т.е. не может быть удален из (2) с помощью калибровочного преобразования (тождественного на базе преобразования накрытия). В соответствии c $[15$, Secs. 3.2, 3.6], $[7,8,20]$ (см. также [6]), для доказательства этого утверждения достаточно заметить, что симметрия $\psi_{4}$ не может быть продолжена до симметрии системы (2). Тогда для векторного поля $V=\frac{1}{2} z \partial_{x}+t \partial_{z}$, соответствующего генератору $\psi_{4}$, действие преобразования $\exp (2 \lambda V)$ на форму Уолквиста-Эстабрука

$$
\omega=d q-u_{z} q_{y} d t-q_{x} d x-q_{y} d y-u_{x} q_{y} d z,
$$

которая определяет накрытие (2) с $\lambda=0$, имеет вид

$$
e^{2 \lambda V} \omega=d q-\left(\lambda^{2} q_{x}+\left(\lambda u_{x}+u_{z}\right) q_{x}\right) d t-q_{x} d x-q_{y} d y-\left(\lambda q_{x}+u_{x} q_{y}\right) d z .
$$

Эта форма является формой Уолквиста-Эстабрука накрытия (2) с $\lambda \in \mathbb{R}$.

5. Тени и оператор рекурсии. Для построения оператора рекурсии уравнения (1) используем метод статьи [27] (см. также $[1,9,17,21,25])$. Найдем тени вида $\Phi\left(t, x, y, z, u, u_{t}, u_{x}, u_{y}, u_{z}, q, q_{x}, q_{y}\right)$ в накрытии (2). В результате прямых вычислений получим тени

$$
q_{x} q_{y}^{-1}, \quad\left(x+\lambda z+\lambda^{2} t\right) q_{x} q_{y}^{-1}+t\left(\lambda u_{x}-u_{z}\right), \quad H\left(q, x+\lambda z+\lambda^{2} t\right) q_{y}^{-1},
$$

где $H$-произвольная функция двух аргументов. Чтобы найти оператор рекурсии, достаточно рассмотреть тень $s=q_{x} q_{y}^{-1}$. Дифференцируя оба уравнения системы $(2)$ по $x$ и подставляя 
$q_{x}=s q_{y}$, получим другое накрытие

$$
\left\{\begin{array}{l}
s_{t}=\lambda s_{z}+u_{z} s_{y}-u_{y z} s+u_{x z} \\
s_{z}=\lambda s_{x}+u_{x} s_{y}-u_{x y} s+u_{x x}
\end{array}\right.
$$

для уравнения (1). Отметим, что $s$ является решением линеаризации (7) уравнения (1). Разложим $s$ в ряд Лорана по $\lambda$ :

$$
s=\sum_{n=-\infty}^{+\infty} \lambda^{n} s_{n}
$$

Так как уравнение $(7)$ не зависит от $\lambda$, каждый коэффициент $s_{n}$ этого ряда является решением $(7)$. Подставляя (11) в (10), получим

$$
\left\{\begin{array}{l}
s_{n+1, t}=s_{n, z}+u_{z} s_{n+1, y}-u_{y z} s_{n+1}+u_{x z} \\
s_{n+1, z}=s_{n, x}+u_{x} s_{n+1, y}-u_{x y} s_{n+1}+u_{x x} .
\end{array}\right.
$$

Вводя обозначения $s_{n+1}=\Psi, s_{n}=\Phi+\psi_{6}$, имеем

$$
\left\{\begin{array}{l}
\widetilde{D}_{t}(\Psi)=\widetilde{D}_{z}(\Phi)+u_{z} \widetilde{D}_{y}(\Psi)-u_{y z} \Psi \\
\widetilde{D}_{z}(\Psi)=\widetilde{D}_{x}(\Phi)+u_{x} \widetilde{D}_{y}(\Psi)-u_{x y} \Psi
\end{array}\right.
$$

Эта система определяет оператор рекурсии $\Psi=\mathcal{R}_{+}(\Phi)$ для симметрий уравнения $(1)$. Обратный оператор $\Phi=\mathcal{R}_{-}(\Psi)$ задается системой

$$
\left\{\begin{array}{l}
\widetilde{D}_{x}(\Phi)=\widetilde{D}_{z}(\Psi)-u_{x} \widetilde{D}_{y}(\Psi)+u_{x y} \Psi \\
\widetilde{D}_{z}(\Phi)=\widetilde{D}_{t}(\Psi)-u_{z} \widetilde{D}_{y}(\Psi)+u_{y z} \Psi
\end{array}\right.
$$

Отметим, что в случае локальных функций $\Phi$ и $\Psi$ расширенные полные производные $\widetilde{D}_{x^{i}}$ в системах (12), (13) могут быть заменены на полные производные $D_{x^{i}}$.

6. Действие оператора рекурсии на локальные симметрии. Действие операторов $\mathcal{R}_{+}$, $\mathcal{R}_{-}$на локальные симметрии уравнения (1) представлено в следующей таблице:

\begin{tabular}{|c|c|c|}
\hline$\Phi$ & $\mathcal{R}_{+}(\Phi)$ & $\mathcal{R}_{-}(\Phi)$ \\
\hline$\phi_{0}(A)$ & $-\phi_{1}(A)$ & $\zeta(A)$ \\
\hline$\phi_{1}(A)$ & 0 & $-\phi_{0}(A)$ \\
\hline$\psi_{1}$ & $r_{1}-t u_{z}$ & $2 w_{1}+t w_{2}+x u_{z}-u u_{y}$ \\
\hline$\psi_{2}$ & $\psi_{5}$ & $w_{2}$ \\
\hline$\psi_{3}$ & $-2 r_{1}-z u_{x}$ & $-2 w_{1}-z u_{t}+u u_{y}-2 x u_{z}$ \\
\hline$\psi_{4}$ & $\frac{1}{2} r_{2}-t u_{x}$ & $\frac{1}{2} w_{3}-t u_{t}-\frac{1}{2} z u_{x} u_{y}-u$ \\
\hline$\psi_{5}$ & $\psi_{6}$ & $\psi_{2}$ \\
\hline$\psi_{6}$ & $r_{3}$ & $\psi_{5}$ \\
\hline
\end{tabular}

Здесь

$$
\zeta(A)=A\left(w_{4}-\frac{1}{2} u_{y}^{2}\right)-A_{t}\left(z u_{y}+x\right)-A_{y}\left(w_{1}-u u_{y}\right)-\frac{1}{2} A_{t t} z^{2}+A_{t y} z u-\frac{1}{2} A_{y y} u^{2}
$$


и $r_{1}, r_{2}, r_{3}, w_{1}, \ldots, w_{4}$ являются решениями систем

$$
\begin{aligned}
& \left\{\begin{array}{l}
r_{1, t}=u_{z} r_{1, y}-u_{y z} r_{1}+x u_{x z}, \\
r_{1, z}=u_{x} r_{1, y}-u_{x y} r_{1}+x u_{x x},
\end{array}\right. \\
& \left\{\begin{array}{l}
r_{2, t}=u_{z} r_{2, y}-u_{y z} r_{2}+u_{x}-z u_{x z}, \\
r_{2, z}=u_{x} r_{2, y}-u_{x y} r_{2}-z u_{x x},
\end{array}\right. \\
& \left\{\begin{array}{l}
r_{3, t}=u_{z} r_{3, y}-u_{y z} r_{3}-u_{x z}, \\
r_{3, z}=u_{x} r_{3, y}-u_{x y} r_{3}-u_{x x},
\end{array}\right. \\
& \left\{\begin{array}{l}
w_{1, x}=u_{x} u_{y}-u_{z}, \\
w_{1, z}=u_{y} u_{z}-u_{t},
\end{array}\right. \\
& \left\{\begin{array}{l}
w_{2, x}=u_{x} u_{t y}-u_{t} u_{x y}-u_{t z}, \\
w_{2, z}=u_{z} u_{t y}-u_{t} u_{y z}-u_{t t},
\end{array}\right. \\
& \left\{\begin{array}{l}
w_{3, x}=z\left(u_{y} u_{x x}+u_{x} u_{x y}-u_{x z}\right)+u_{x}, \\
w_{3, z}=z\left(u_{y} u_{x z}+u_{z} u_{x y}-u_{t x}\right)+u_{x} u_{y},
\end{array}\right. \\
& \left\{\begin{array}{l}
w_{4, x}=u_{x} u_{y y}-u_{y z}, \\
w_{4, z}=u_{z} u_{y y}-u_{t y} .
\end{array}\right.
\end{aligned}
$$

Отметим, что системы (17)-(20) задают абелевы накрытия, или, другими словами, $w_{1}, \ldots, w_{4}$ представляют собой потенциалы двухкомпонентных законов сохранения уравнения (1), в то время как системы (14) и (15) определяют новые неабелевы накрытия этого уравнения. Функция $r_{3}$ отличается знаком от решения системы (10) с $\lambda=0$. Отметим также, что в отличие от уравнения (1) системы (14), (15) и (19) не инвариантны относительно трансляций с генераторами $\partial_{x}$, $\partial_{z}, \partial_{z}$ соответственно. Примеры таких трансляционно не инвариантных накрытий обсуждались в [23], где было показано их происхождение из нетривиальных экзотических 2-коциклов.

7. Заключение. В статье была рассмотрена структура алгебры локальных симметрий четырехмерного уравнения универсальной иерархии (1) и построены операторы рекурсии для него. Мы показали, что действие операторов рекурсии на некоторые локальные симметрии дает новые неабелевы накрытия этого уравнения. Некоторые из этих новых накрытий оказываются не инвариантными относительно трансляций независимых переменных. Мы показали нетривиальность второй группы экзотических когомологий алгебры симметрий уравнения (1). Важной задачей для дальнейшего изучения является установление связей между соответствующими неабелевыми расширениями этой алгебры Ли и свойствами интегрируемости уравнения (1) (см. [22-24]). Также интересной задачей представляется изучение нелокальных симметрий и нелокальных законов сохранения в накрытиях, ассоциированных с (2) (см. $[1,10,16])$.

\section{СПИСОК ЛИТЕРАТУРЫ}

1. Баран Х., Красильщик И. С., Морозов О. И., Войчак П. Нелокальные симметрии интегрируемых линейно вырожденных уравнений: сравнительное исследование// Теор. мат. физ. - 2018. - 196 (2). - C. 169-192.

2. Виноградов А. М., Красильщик И. С. Симметрии и законы сохранения дифференциальных уравнений математической физики. - М.: Факториал, 2005.

3. Мартинез Алонсо Л., Шабат А. Б. Гидродинамические редукции и решения универсальной иерархии // Теор. мат. физ. - 2004. - 140 (2). - С. 216-229.

4. Baran H., Marvan M. Jets: A software for differential calculus on jet spaces and diffieties. http://jets.math.slu.cz.

5. Bogdanov L. V., Pavlov M. V. Linearly degenerate hierarchies of quasiclassical SDYM type// J. Math. Phys. - 2017. - 58 (9). - 093505.

6. Cieśliński J. Group interpretation of the spectral parameter in the case of nonhomogeneous, nonlinear Schrödinger system// J. Math. Phys. — 1993. - 34 (6). — P. 2372-2384. 
7. Igonin S., Krasil'shchik J. On one-parametric families of Bäcklund transformations/arXiv: nlin/0010040 [nlin.SI].

8. Krasil'shchik J. On one-parametric families of Bäcklund transformations/ Preprint DIPS-1/2000.

9. Kruglikov B. S., Morozov O. I. Integrable dispersionless PDEs in 4D, their symmetry pseudogroups and deformations// Lett. Math. Phys. - 2015. - 105. — P. 1703-1723.

10. Krasil'shchik I. S., Sergyeyev A., Morozov O. I. Infinitely many nonlocal conservation laws for the ABC equation with $A+B+C \neq 0 / /$ Calc. Var. Part. Diff. Eqs. - 2016. - 55, № 5. - 123.

11. Krasil'shchik J., Verbovetsky A. Geometry of jet spaces and integrable systems// J. Geom. Phys. — 2011. - 61. - P. 1633-1674.

12. Krasil'shchik J., Verbovetsky A., Vitolo R. A unified approach to computation of integrable structures// Acta Appl. Math. - 2012. - 120. - P. 199-218.

13. Krasil'shchik J., Verbovetsky A., Vitolo R. The Symbolic Computation of Integrability Structures for Partial Differential Equations. - Springer-Verlag, 2017.

14. Krasil'shchik I. S., Vinogradov A. M. Nonlocal symmetries and the theory of coverings// Acta Appl. Math. - 1984. - 2. - P. 79-86.

15. Krasil'shchik I. S., Vinogradov A. M. Nonlocal trends in the geometry of differential equations: symmetries, conservation laws, and Bäcklund transformations// Acta Appl. Math. - 1989. — 15. — P. 161-209.

16. Lelito A., Morozov O. I. Three-component nonlocal conservation laws for Lax-integrable 3D partial differential equations// J. Geom. Phys. - 2018. - 131. - P. 89-100.

17. Malykh A. A., Nutku Y., Sheftel M. B. Partner symmetries of the complex Monge-Ampère equation yield hyper-Kähler metrics without continuous symmetries// J. Phys. A. - 2003. - 36. - 10023.

18. Martinez Alonso L., Shabat A. B. Energy-dependent potentials revisited: A universal hierarchy of hydrodynamic type// Phys. Lett. A. - 2002. - 299. - P. 359-365.

19. Marvan M. Another look on recursion operators// in: Differential Geometry and Applications/ Proc. Conf. - Brno: Masaryk University, 1995. - P. 393-402.

20. Marvan M. On the horizontal gauge cohomology and nonremovability of the spectral parameter// Acta Appl. Math. - 2002. - 72. - P. 51-65.

21. Marvan M., Sergyeyev A. Recursion operators for dispersionless integrable systems in any dimension// Inv. Probl. - 2012. - 28. - 025011.

22. Morozov O. I. Deformed cohomologies of symmetry pseudo-groups and coverings of differential equations// J. Geom. Phys. - 2017. — 113. - P. 215-225.

23. Morozov O. I. Deformations of infinite-dimensional Lie algebras, exotic cohomology, and integrable nonlinear partial differential equations// J. Geom. Phys. - 2018. - 128. - P. 20-31.

24. Morozov O. I. Deformations of infinite-dimensional Lie algebras, exotic cohomology, and integrable nonlinear partial differential equations. II/ arXiv: 1805.00319 [nlin.SI].

25. Morozov O. I., Sergyeyev A. The four-dimensional Martínez Alonso-Shabat equation: reductions and nonlocal symmetries// J. Geom. Phys. - 2014. - 85. - P. 40-45.

26. Pavlov M. V., Stoilov N. Three dimensional reductions of four-dimensional quasilinear systems// J. Math. Phys. - 2017. - 58 (11). - 111510.

27. Sergyeyev A. A simple construction of recursion operators for multidimensional dispersionless integrable systems// J. Math. Anal. Appl. — 2017. - 454. - P. 468-480.

Морозов Олег Игоревич

Институт проблем управления им. В. А. Трапезникова РАН, Москва;

Факультет прикладной математики, Университет AGH, Краков, Польша

E-mail: morozov@agh.edu.pl 